\title{
Técnicas para el diagnóstico de transformadores de potencia: Una revisión crítica
}

\author{
Techniques for power transformers diagnosis: A critical review
}

\author{
Juan David Zorrilla Henao ${ }^{1} \quad$ Alexander Céspedes Fernández ${ }^{1 *}$ \\ Diego Fernando García Gómez ${ }^{1}$
}

Recibido 26 de abril de 2018, aceptado 31 de enero de 2019
Received: April 26, 2018 Accepted: January 31, 2019

\begin{abstract}
RESUMEN
Los sistemas de transmisión de energía eléctrica comprenden un enlace fundamental en el sector productivo y social de un país. Uno de los principales componentes de estos sistemas son los transformadores de potencia que permiten la exportación de energía en alta tensión a grandes distancias. La falla de un transformador de potencia puede conllevar el colapso parcial o total de un sistema eléctrico. Existen diversas técnicas para el mantenimiento y diagnóstico de dichos equipos. En este trabajo se realiza una revisión crítica de ocho de las principales técnicas de diagnóstico de transformadores de potencia incluyendo una descripción básica y evolución histórica, teniendo en cuenta que actualmente son aplicadas en línea o que tienen un alto potencial para llegar a serlo.
\end{abstract}

Palabras clave: Técnicas de diagnóstico en línea, monitoreo, transformadores de potencia, revisión crítica.

\begin{abstract}
Power transmission systems involve an essential relationship in the productive and social sectors of a country. The power transformers are one of the main components in the power systems; these systems allow exporting energy in high voltage over long distances. Failure of a power transformer can lead to a partial or total collapse of an electrical system. There are several procedures for maintenance and diagnosis of such equipment. In this paper, a critical review of eight of the main diagnostic techniques of power transformers is made, including its description and historical evolution, taking into account that currently, those methods can be applied online or those that probably will be applied.
\end{abstract}

Keywords: On-line diagnostic techniques, monitoring, power transformers, critical review

\section{INTRODUCCIÓN}

Los transformadores de potencia son elementos fundamentales para la transmisión de energía eléctrica desde los centros de generación hasta los centros de consumo, por esta razón, conocer su estado operativo es de vital importancia para garantizar un funcionamiento fiable y libre de fallas. Adicionalmente, la sustitución de dichos activos después de una falla súbita o mantenimiento correctivo, a menudo no es una alternativa económicamente óptima, ya que los costos asociados a esta pueden ser enormes y muchos de los modos de falla dependen de las condiciones de trabajo de cada transformador.

\footnotetext{
1 Universidad del Valle. Facultad de Ingeniería. Cali, Colombia.

E-mail: juan.zorrilla@correounivalle.edu.co; alexander.cespedes@correounivalle.edu.co; diego.garcia@correounivalle.edu.co

* Autor de correspondencia: alexander.cespedes@ correounivalle.edu.co
} 
Entre las opciones más atractivas para solucionar este inconveniente, se encuentra el uso de pruebas de diagnóstico y sistemas de monitoreo en línea, con el fin de averiguar el estado del transformador en operación, y aún más, cuando están ubicados en lugares remotos o puntos neurálgicos de la red eléctrica.

En este trabajo se realiza una revisión crítica de algunas técnicas de diagnóstico utilizadas en los transformadores de potencia, describiendo sus principales características, su desarrollo evolutivo y proponiendo un análisis comparativo de las mismas, centrándose en aquellas técnicas con alto potencial para ser aplicadas en línea o eventualmente que ya estén siendo realizadas de esta manera. Finalmente, se muestran las respectivas conclusiones y recomendaciones del caso.

\section{TÉCNICAS DE DIAGNÓSTICO DE TRANSFORMADORES DE POTENCIA: BREVE DESCRIPCIÓN E HISTORIA}

Las fallas en un transformador generalmente poseen orígenes distintos y mezclados entre sí, es decir, que varias causas pueden crear un solo efecto [1]. Entre las posibles causas de falla se tienen: errores provenientes del diseño o fabricación, daño en los equipos auxiliares del transformador, error humano durante los procesos de mantenimiento y operación de los equipos y fallas en los circuitos de protección. Por otra parte, existen fallas progresivas, tales como: deformación de los materiales de aislamiento interno y de los devanados, deterioro del aislamiento externo debido a condiciones medioambientales como viento, nieve, sal o polvo o daños en la instrumentación que eviten detectar el deterioro gradual debido a fugas de aceite, fugas de gas, etc. Debido a lo anterior, las técnicas de diagnóstico de fallas en transformadores de potencia adquieren vital relevancia. A continuación se describen brevemente cada una de las ocho técnicas de diagnóstico seleccionadas y adicionalmente se realiza un corto recorrido por su historia.

\section{Técnica de Análisis de Gases Disueltos (DGA)}

La técnica de Análisis de Gases Disueltos, es un método que tiene como finalidad analizar los gases generados por fallas incipientes en transformadores sumergidos en aceite. A medida que el transformador se ve sometido a esfuerzos eléctricos y mecánicos, se van generando los gases que son resultado de la descomposición de los materiales aislantes, papel y aceite [2], ocasionando cambios inmediatos y observables que incluso pueden determinar fallas en proceso de evolución. El diagnóstico a través de DGA considera un nivel mínimo admisible para cada uno de estos gases y el cual está establecido en normas como la IEEE C57.146 [3] e IEEE C57.104 [4]. Diferentes combinaciones entre gases permiten inferir problemas de tipo térmico o eléctrico en el transformador [5].

\section{Breve historia}

El análisis de Gases disueltos inicia en 1960 y es utilizado ampliamente en el mundo para detectar averías en los transformadores, diez años después aparecen diferentes técnicas de interpretación tales como Dornenburg (1970), Gases Clave (1973-74), Técnica de Duval (1974) y Rogers (1978). Para el año de 1991 el Institute of Electrical and Electronics Engineers (IEEE) publica el estándar C57.104 con su última revisión en el año 2008 [3]. En 1999 la International Electrotechnical Commission (IEC) publica su guía para interpretar los gases disueltos llamada IEC 60599 con algunas mejoras publicadas en 2007 y la última revisión oficial en 2015 [6]. En 2002 Michel Duval publica dos artículos, el primero es una interpretación de DGA utilizando la IEC 60599 y comparándolo con la base de datos del comité técnico TC-10 (Comité Técnico de Fluidos para aplicaciones electrotécnicas) de la IEC en compañía de A. dePablo [7]. El segundo, es una revisión de las fallas detectables por análisis de gases en el aceite en los transformadores a través del triángulo de Duval, haciendo énfasis en la interpretación de la prueba [8]. En el año 2004 Sayed A. Ward implementa DGA como diagnóstico para evaluar la condición de los transformadores en servicio [9] y un año más tarde Michel Duval y James Dukarm proponen algunas mejoras para DGA [10]. Para 2006 Suwarmo reporta datos experimentales sobre los efectos del arco en los gases disueltos en los aceites de los transformadores [11], dos años después, Michel Duval propone nuevas versiones de su triángulo para interpretar diferentes tipos de fallas [12] y en 2009 se publica una nueva versión de las guías IEEE anteriormente nombradas y de la American Society for Testing Materials ASTM D 3612 [13]. En el año 2010 aparece una publicación de A. Mackenzie donde muestra como el DGA puede ser usado para realizar monitoreo en línea [14]. Luego, en el año 2011 se utilizan las redes neuronales artificiales con el fin de realizar interpretaciones de averías a través de DGA, trabajo 
realizado por M. Golkhah [15]. Los dos años siguientes se realizan diferentes artículos de correlaciones entre interpretaciones de resultados de DGA destacándose la publicación de H.C. Yung [16]. En el año 2014, Michel Duval publica una nueva forma gráfica de interpretar DGA a través de un pentágono [17-18].

En la Figura 1 se puede observar un ejemplo del pentágono propuesto que considera 5 gases primarios los cuales son: Hidrógeno $\left(\mathrm{H}_{2}\right)$, Metano $\left(\mathrm{CH}_{4}\right)$, Etano $\left(\mathrm{C}_{2} \mathrm{H}_{6}\right)$, Etileno $\left(\mathrm{C}_{2} \mathrm{H}_{4}\right)$ y Acetileno $\left(\mathrm{C}_{2} \mathrm{H}_{2}\right)$, en donde el porcentaje representativo de cada uno de estos, permite calcular a través de un método geométrico basado en las coordenadas que conforman el centroide del pentágono, las posibles zonas de falla presentes en el transformador. Se puede identificar una zona de falla por descargas parciales (PD), una zona por descargas de baja energía (D1), una zona por descargas de alta energía (D2), una zona por fallas térmicas mayores a $700{ }^{\circ} \mathrm{C}(\mathrm{T} 3)$, una zona por fallas térmicas entre $300{ }^{\circ} \mathrm{C}$ y $700{ }^{\circ} \mathrm{C}$ (T2), y una zona por fallas térmicas menores a $700{ }^{\circ} \mathrm{C}$ (T3), entre otros tipo de fallas detectables.

\section{ANÁLISIS DE RESPUESTA EN FRECUENCIA (FRA)}

El método de Análisis de Respuesta en Frecuencia básicamente es utilizado para verificar la integridad mecánica de los núcleos de transformador, los devanados y los bastidores de presión de los transformadores de potencia. Consiste en la

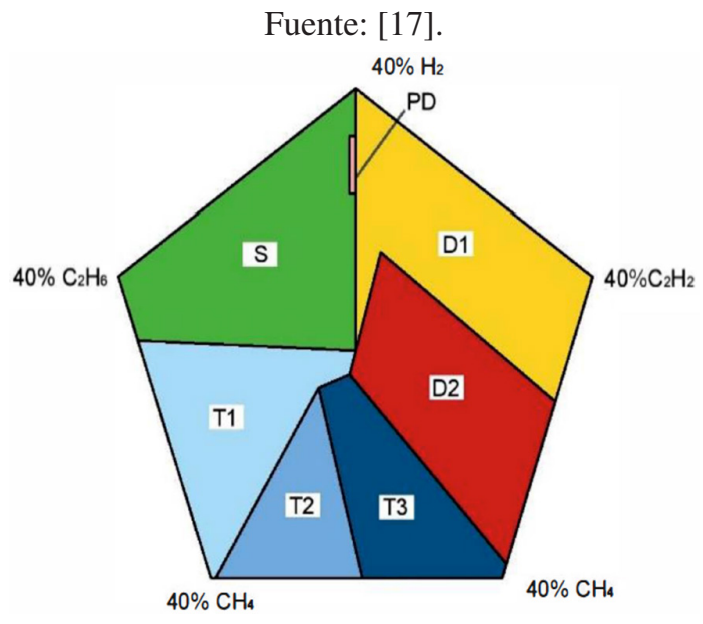

Figura 1. Pentágono de Duval. medición de la relación entre la señal de respuesta en un transformador ante un estímulo, para un amplio rango de frecuencia y la comparación con mediciones de referencia o fábrica [19].

La medición puede ser realizada con 3 métodos diferentes: IFRA (Impulse Frequency Response Analysis) se inyecta un impulso de tensión a los devanados [20], SFRA (Sweep Frequency Response Analysis) se hace un barrido de frecuencia utilizando una señal de tensión sinusoidal [21] o PRBS (Pseudo Random Binary Sequence) se utilizan señales con algoritmos deterministas y aleatorios para generar los espectros de frecuencia. Este ensayo de respuesta en frecuencia tiene como metodología, medir la magnitud de la impedancia, admitancia y magnitudes de corriente en los devanados de los transformadores en una gama de frecuencias comprendidas generalmente entre los $10 \mathrm{~Hz}$ y $3 \mathrm{MHz}$.

La medición para los transformadores en buen estado o recién fabricados puede suministrar una especie de huella digital o diagrama de referencia, con la que se comparará el equipo, posterior a la ocurrencia de una falla o evento que pueda haber causado daños en el mismo [22-23]. La normatividad respecto a FRA ha sido difundida a través de instituciones como IEC, ICS [24] y CIGRÉ [25]. La Tabla 1 muestra los principales tipos de falla que pueden ser detectados utilizando la técnica de FRA.

\section{Breve historia}

El análisis de respuesta en frecuencia, tuvo su iniciación en los transformadores en el año 1978 por parte de los ingenieros E.P. Dick y Clifford C. Erven explicando cómo se podría realizar FRA [26], y las ventajas que tenía para la determinación de los parámetros internos del transformador, hablando específicamente de los devanados. Diecisiete años después aparecen J.A. Lapworth y J. Noonan presentando una descripción detallada de FRA y realizan una nueva publicación como actualización en 2007 usando el método SFRA que básicamente consiste en inyectar ondas sinusoidales durante la prueba [27].

En 2002, la IEEE recomienda un método con tres componentes principales, fuente, referencia y medida. Lo anterior, debido a un acuerdo entre la comunidad científica, el cual es acatado por todas las compañías fabricantes de instrumentos 
Tabla 1. Resumen del tipo de fallas detectadas en transformadores utilizando FRA [20].

\begin{tabular}{|c|l|l|}
\hline \multicolumn{1}{|c|}{ Ítem } & \multicolumn{1}{|c|}{ Naturaleza del defecto } & \multicolumn{1}{|c|}{ Detectabilidad } \\
\hline 1 & Conexión a tierra defectuosa de núcleo & Detectable \\
\hline 2 & Pérdida de presión de torque en los devanados & Usualmente no detectable \\
\hline 3 & Movimiento del núcleo & Detectable (si es muy severo) \\
\hline 4 & Envejecimiento convencional & Detectable (si es muy severo) \\
\hline 5 & Envejecimiento convencional & Detectable \\
\hline 6 & Espiral corto-circuitadas o abiertas & Detectable \\
\hline 7 & Estructuras de sujeción rotas & Detectable \\
\hline 8 & Espiras flojas & Detectable \\
\hline 9 & Colapso parcial de los devanados & Detectable \\
\hline 10 & Pandeo de flejes de fijación & Detectable \\
\hline 11 & Deformación del devanado circunferencial, inclinación en espiral & Detectable \\
\hline
\end{tabular}

de prueba para SFRA, publicando en el año 2013 el estándar C.57-149 [28]. En el caso de China, también se publicó una norma conocida como DL 911 de 2004 con una última revisión en el año 2016, dicho estándar solo hace referencia a medidas usando SFRA e incluye el principio del método, requerimientos para ejecutarlo y sugiere métodos básicos para el análisis de resultados [29]. En 2003 IEC crea el comité de estudio SC-A2Transformadores, fundando un grupo de trabajo para la aplicación de FRA en transformadores de potencia publicando la norma IEC 60076-18 [30]. Además se destacan otros artículos que siguen contribuyendo en la evolución del método [31-37]. En Colombia también se han realizado publicaciones relacionadas con esta temática [38-41].

\section{MEDICIÓN DE CORRIENTES DE POLARIZACIÓN Y DESPOLARIZACIÓN (PDC)}

El análisis PDC es un método de ensayo dieléctrico no invasivo ni destructivo que sirve para determinar la conductividad y el contenido de humedad de los materiales aislantes en un transformador [42]. La medición PDC se realiza mediante la aplicación de una tensión de DC a través del objeto de prueba durante un tiempo determinado, en un rango que puede ir desde 5 minutos hasta 3 horas dependiendo de las cantidad de puntos de muestreo que se requieran tomar durante la prueba. A continuación, la fuente de tensión de DC se desconecta y el objeto de prueba se cortocircuita. El proceso de polarización activado previamente da lugar a una corriente de descarga en la dirección opuesta, donde no está presente ninguna contribución de la conductividad, es decir, la corriente de despolarización [43], este fenómeno puede es resumido en la Figura 2, donde $T_{P}$ y $T_{D}$ son los tiempos de polarización y despolarización respectivamente y $U_{0}$ corresponde a la magnitud de tensión de polarización aplicada. Las corrientes de carga $\left(i_{P O L}\right)$ y descarga $\left(i_{D E P O L}\right)$ están influenciadas por las propiedades del material aislante, así como por su estructura geométrica.

\section{Breve historia}

El método PDC es oficializado en 1991 con la publicación de la guía de la IEEE C57.106 [4446], en esta se estipulan los límites de contenido de humedad en los transformadores según su nivel de tensión, Otra normatividad asociada como la IEC 60422 [47], la IEEE 62 de 1995 [48] luego integrada en la norma IEEE C57.152-2013 [49] y

Fuente: [43].

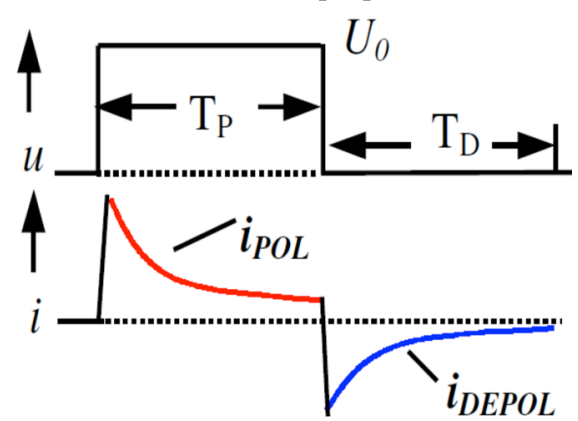

Figura 2. Corrientes de polarización y despolarización en la técnica PDC. 
el reporte 349 del CIGRÉ del 2008 [50]; también son utilizadas en el desarrollo e investigación del método PDC e incluso en los otros métodos que evalúan la respuesta dieléctrica del aislamiento. En 1998 V.D. Houhanessian y W.S. Zaengl publican un artículo donde explican el modelo circuital que existe del aislamiento aceite-papel del transformador [51].

En 2002 T. Leibfried y A.J. Kachler realizan una descripción detallada del método PDC para utilizarlo en línea [43], teniendo en cuenta una publicación anterior difundida en el año 2000 por J.J. Allf, V.D. Houhanessian, W.S. Zaengl y A.J. Kachler [52]. En los siguientes 11 años, se publican artículos relacionados con la interpretación del espectro de polarización, la correlación con otras técnicas de análisis dieléctrico y desarrollos en software relacionados y con la mejora en la ejecución del método [53-59]. Para el año 2014 S.A. Bhumiwat realiza una nueva entrega investigativa sobre la conducción inusual de corriente en la interfaz papel-aceite por PDC explicando que algunos contaminantes pueden producir ciertas descargas de energía en dichos elementos [60]. En 2015 M.A. Talib, N.A. Muhamad y Z.A. Malek realizan una clasificación de las fallas usando PDC y explicando los límites de corrientes y constantes de tiempo para fallas como arcos, sobrecalentamiento y descargas parciales [61].

\section{ESPECTROSCOPIA EN EL DOMINIO DE LA FRECUENCIA (FDS)}

El método de diagnóstico FDS, es de tipo no invasivo que se encarga de evaluar la condición del aislamiento aceite-papel en el transformador, basado en la aplicación de una tensión sinusoidal a través de los terminales del objeto de prueba, midiendo la amplitud y fase de la corriente de respuesta que fluye a través de aislamiento con el fin de determinar los parámetros capacitivos del sistema aislante [62]. Generalmente, esta prueba va acompañada de PDC debido a los rangos de frecuencia manejados por ambas, PDC para bajas frecuencias y FDS para altas y permite detectar fallas tales como pérdidas, capacitancia y nivel de humedad en el aislamiento celulósico [62-63]. También se puede asumir como una extensión de las mediciones de factor de disipación (tanঠ) realizadas a frecuencia nominal. El brochure 414 de CIGRÉ y otros manuales son una guía que explica la interpretación de FDS [64, 65]. Además, este método se realiza bajo los límites que proporcionan las normas internacionales tales como la IEC 61620 de 1998 [66]. La Figura 3 muestra los distintos factores que influyen en la técnica de FDS.

\section{Breve historia}

En el año 2000 se presenta el método FDS como una posible técnica de diagnóstico de transformadores de potencia por U. Gäfbert y L. Adeen, entre otros [67].

\section{Fuente: [65].}

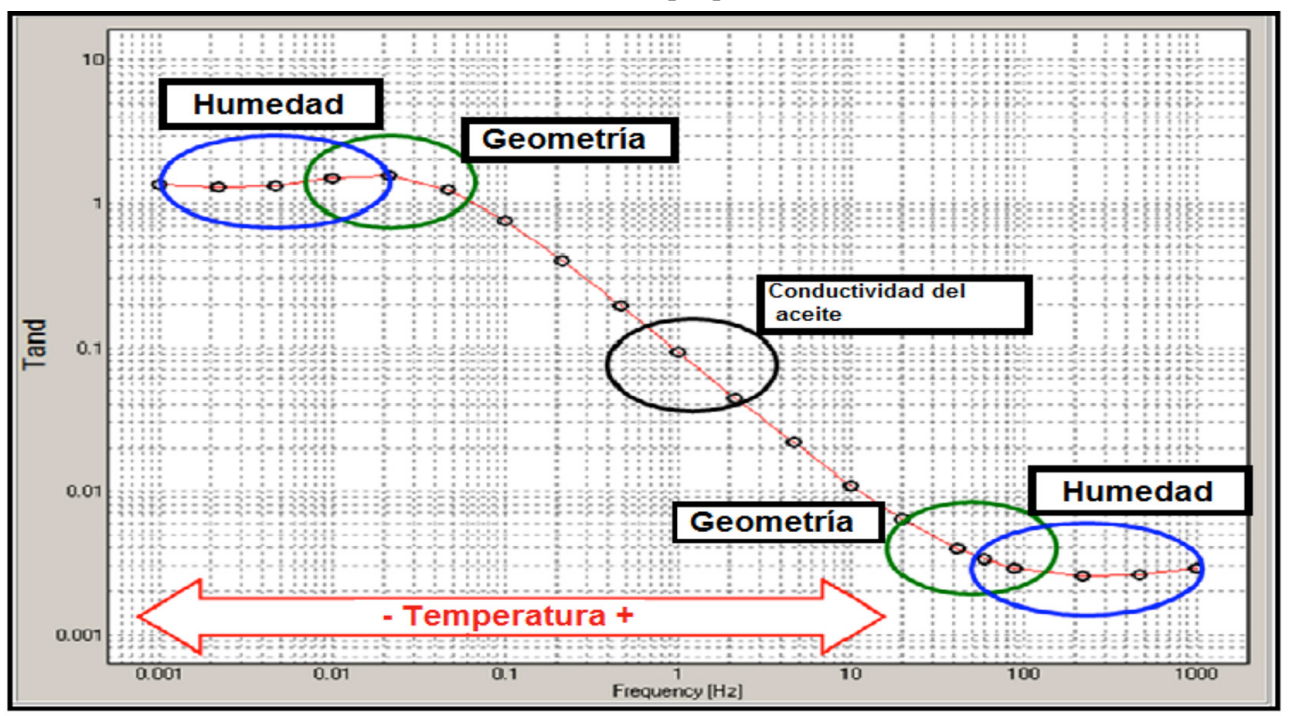

Figura 3. Influencia de factores físico-químicos del transformador en la técnica de FDS. 
En 2002 el grupo de trabajo 15.01 de CIGRÉ, publica una guía sobre métodos de respuesta dieléctrica para el diagnóstico de transformadores de potencia, en donde es considerado como un buen complemento a los métodos utilizados hasta el momento (PDCRVM) para determinar la condición de humedad del aislamiento celulósico [68]. Un año después W. Zaengl hace publicaciones relacionadas con la aplicabilidad de la espectroscopia en el dominio del tiempo y frecuencia para equipos de alta tensión [69-70].

Adicionalmente, artículos publicados por T. Saha y P. Purkait [71], Gobi K. Supramaniam [72], A. Bouaicha [73], I. Fofana [74], y W. Wang [75], han sido fundamentales para obtener nuevos desarrollos de esta prueba. En el año 2012 M.K. Pradhan realiza una investigación experimental sobre los parámetros que afectan la evaluación de la condición de aislamiento usando FDS [76]; y R. Liao y otros, hacen un análisis cuantitativo de la condición de envejecimiento del aislamiento aceite papel por FDS [77]. También M. Jaya, Y. Rink, y T. Leibfried publican respecto a esta temática [78]. En el año 2016 se publican dos artículos destacados: el primero plantea utilizar el método de FDS en muy bajas frecuencias [79] por Y. Liu, T. Zhang; y el segundo utilizando aceite con nanopartículas por J. Dai, M. Dong, Y. li y otros [80].

\section{MEDICIÓN DE LA TENSIÓN DE RECUPERACIÓN (RVM)}

En la técnica de RVM, la función principal es determinar el estado del aislamiento aceite-papel de un transformador. Por lo general, es una prueba que puede ser complementaria a la prueba de PDC o FDS. Cuando se aplica una tensión de DC al aislamiento, las moléculas se polarizan y se orientan en dirección del campo eléctrico. Luego, se abre el interruptor de la fuente de DC y a su vez se cierra un segundo interruptor que permite aplicar un cortocircuito entre los terminales del aislamiento bajo prueba por un breve de período de tiempo (por lo general, corresponde a la mitad del tiempo de carga) despolarizando parcialmente las moléculas. Finalmente, la tercera y última etapa ocurre al momento de abrir el cortocircuito, donde aparece una tensión en los terminales del aislamiento debido a la carga remanente. Esta es la tensión de recuperación y depende en gran medida de la humedad en el sistema papel-aceite del transformador [81].

\section{Breve historia}

Esta prueba tuvo sus inicios en la década de los ochenta. En el año 1991 S. Yamanaka, T. Kabeya realizan una aplicación de tensión residual para diagnosticar la degradación en los materiales aislantes. Tres años más tarde se realiza un modelo teórico de varios materiales dieléctricos y se analiza por RVM basado en polarización interfacial [82]; también se estudia sobre la relación existente entre este método y otros métodos de respuesta dieléctrica planteados hasta el momento [83]. En 1998 se realiza un modelo de mediciones dieléctricas en transformadores de potencia incluyendo RVM y elaborado por CIGRÉ en Francia, además G. Csépes y otros, publican los cimientos prácticos sobre dicho método [84]. Luego, se publican varias técnicas y modelos de interpretación alrededor del RVM $[85,86]$, creando incluso sistemas expertos para su ejecución y lectura [87]. En el año 2009, C.K Dwivedi y M.B. Daigavane realizan una evaluación del contenido de humedad en aislamiento papel-aceite utilizando RVM [88]. A partir del año 2010 hasta la actualidad, las publicaciones relacionadas con este tema han sido enfocadas a mejorar la calidad de la medición e interpretación de resultados, incluyendo parámetros en el dominio del tiempo y de la frecuencia [89-90]. También se debe destacar que en el año 2016 se publica un artículo de Z. Ádám y Á. Szirmai [91] donde se intenta modelar la técnica de RVM usando software especializado.

\section{ANÁLISIS DE FURANOS Y GRADO DE POLIMERIZACIÓN}

El aislamiento sólido del transformador está formado por papel, el cual está compuesto por fibras de celulosa. Esta última es un polímero formado, a su vez, por moléculas de glucosa. La cadena promedio del polímero tiene una longitud de 1.000 a 1.200 moléculas de glucosa cuando el papel está nuevo, antes de su instalación en el transformador y su secado en fábrica [5]; una vez realizado este proceso, el papel ya instalado en el transformador contiene entre 800 a 1.000 moléculas de glucosa. La longitud promedio de la cadena de la celulosa se denomina grado de polimerización del papel (DP por sus siglas en inglés). A medida que este envejece se produce una descomposición gradual 
de estas cadenas y por ende la disminución de la rigidez mecánica del papel, esto puede causar fallas al interior del transformador. Además, durante dicho proceso la molécula de la glucosa cambia formando anillos de furano, que suelen migrar hacia el aceite $\mathrm{y}$ a altas temperaturas forman diversos compuestos químicos asociados a fenómenos térmicos, eléctricos o mecánicos en el transformador. El cambio en el contenido furánico es el parámetro más importante para determinar si hay la posibilidad de una falla que amerite atención. En la Tabla 2 se puede observar un resumen con la interpretación de los diferentes compuestos furánicos que se pueden encontrar en el aceite mineral del transformador.

\section{Breve historia}

En el año 1993 IEC publica el estándar 61198, donde se describe el método para determinar furanos y compuestos relacionados explicando también la técnica analítica HPLC [92]. Durante esta década, surgieron las ecuaciones que relacionan el contenido de furanos con el grado de polimerización, y que son propuestas por Chendong en 1991, Pahlavanpour en 1995, Depablo y Shkolnik en 1999. Además, en este último año se publica la guía ASTM D 5837 y que tiene su más reciente revisión en 2015 [93].

Para el año 2000 se desarrollan investigaciones relevantes por parte A. Emsley y X. Xiao, dando como resultado cuatro publicaciones de su estudio [94-97], y luego en el año 2005, se realiza una revisión similar, pero utilizando aislamiento celulósico "térmicamente estabilizado", destacándose una conferencia en San Diego (Estados Unidos) del ingeniero T.V. Ommen. En 2007 CIGRÉ publica su brochure 323 [98] y a partir de ese año, se han venido realizando actualizaciones de las normas internacionales e investigaciones relacionadas [99105]. Al respecto, se destacan las publicaciones de Z. Wang e I. Höhlein en el año 2016 que estudian las medidas de metanol y óxidos de carbono presentes en la degradación del papel, complementando así la medición de furanos [106-110].

\section{MÉTODO TERMOGRÁFICO}

Es un método realizado principalmente por una herramienta conocida como el termógrafo infrarrojo (IRT-Infrared Thermograph), de carácter no invasivo que permite determinar la condición de temperatura, y por ende realizar un diagnóstico térmico externo (sobrecarga, puntos calientes en bujes o conexiones, etc.) de un transformador [111]. Por medio de la termografía pueden detectarse fallas en su etapa temprana tales como oxidación de interruptores de alta tensión, conexiones recalentadas, defectos de aislamiento, puntos calientes en bujes, sobrecarga, etc. En muchas ocasiones, la termografía ayuda a localizar el problema y establecer la gravedad del mismo. La Figura 4 muestra un ejemplo de análisis termográfico para la detección de puntos calientes en un transformador de potencia.

\section{Breve historia}

A mediados del siglo pasado, la termografía en la industria crea la necesidad de hacer una herramienta portable capaz de reproducir el método en un sitio determinado y en 1960 se crea la primera cámara termográfica. Más adelante, en el año de 1992 se desarrolla el primer focal para cámaras termográficas que combinó ciertos avances electrónicos publicados hasta el momento [113].

Tabla 2. Interpretación de compuestos furánicos [65].

\begin{tabular}{|c|l|l|}
\hline Ítem & \multicolumn{1}{|c|}{ Compuesto Funárico } & \multicolumn{1}{c|}{ Interpretación. } \\
\hline 1 & 2 furaldehído & $\begin{array}{l}\text { Sobrecalentamiento general de la celulosa o condición de falla en el } \\
\text { pasado. }\end{array}$ \\
\hline 2 & 2 furil alcohol & $\begin{array}{l}\text { Se forma en presencia de gran contenido de humedad o descomposición } \\
\text { activa del papel. }\end{array}$ \\
\hline 3 & 2 Acetil furano & $\begin{array}{l}\text { No es común. Representa averías por descargas eléctricas o un alto } \\
\text { índice de esfuerzo eléctrico. }\end{array}$ \\
\hline 4 & 5 metilo 2 furaldehído & $\begin{array}{l}\text { Sobrecalentamiento intenso y localizado, supone una falla ocacionada } \\
\text { por un punto caliente a muy alta temperatura. }\end{array}$ \\
\hline 5 & 5 hidroximetilo 2 furaldehído & $\begin{array}{l}\text { Oxidación del aislamiento sólido. Puede crear cortocircuito en los } \\
\text { devanados, el núcleo, entre otros. }\end{array}$ \\
\hline
\end{tabular}




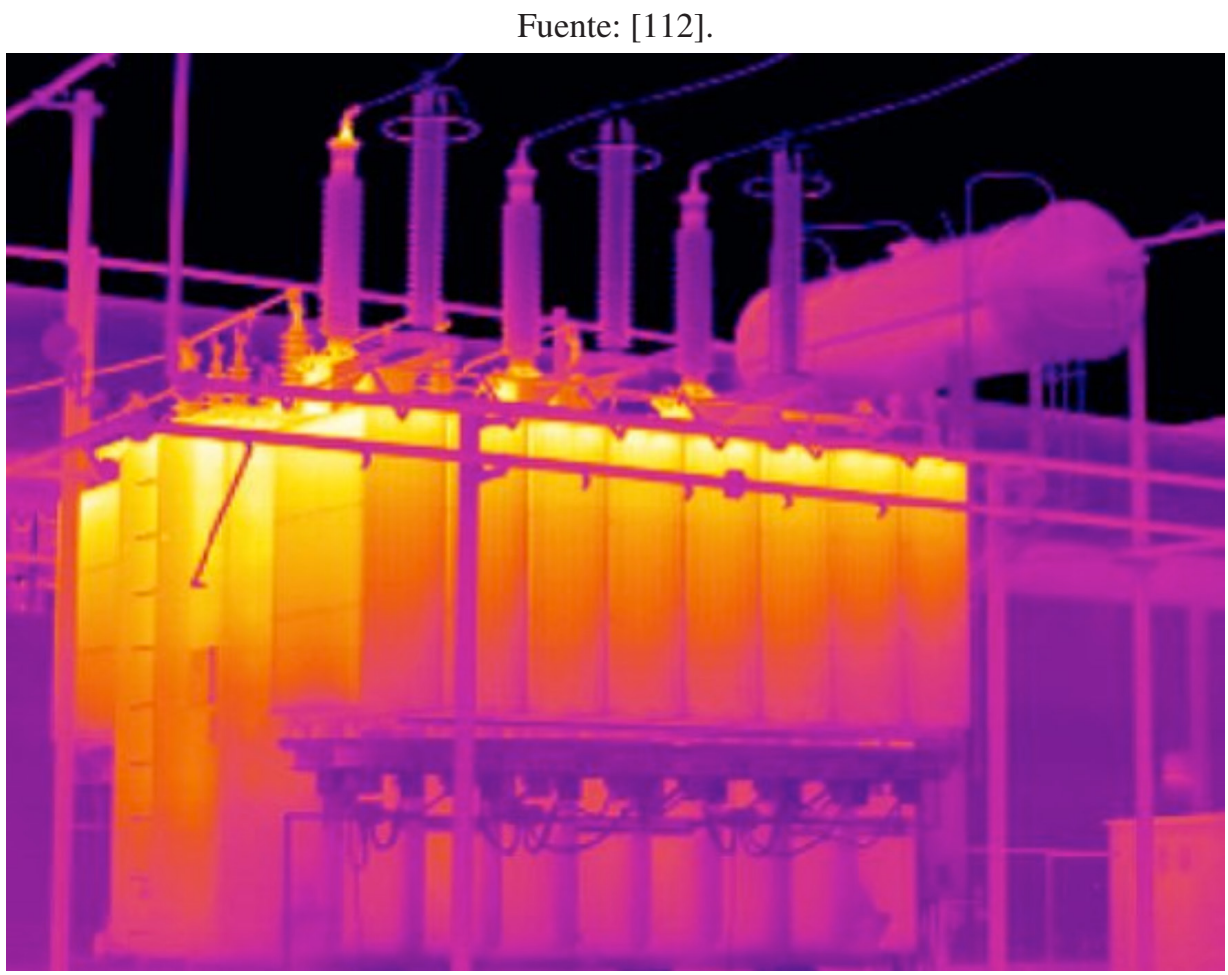

Figura 4. Detección de puntos calientes en un transformador usando IRT.

Entre los avances más notables para la mejora de este método, se tiene el detector QWLP (Quantum Well Infrared Photodetector) creado en 2003 y que es capaz de generar imágenes infrarrojas de muy alta calidad. Estándares IEEE como el C57.134 del año 2013 [114] y el C57.119 del año 2001 [115] que permiten obtener una buena interpretación de las impresiones termográficas que se adquieren durante las pruebas. Por otra parte, se destacan artículos publicados por N. Utami en 2009 [116], y de Q. Khan en 2016 [117], e incluso un manual publicado por la compañía FLIR en 2012 y 2016 [118], convirtiéndose en referentes importantes para esa técnica, utilizada en el mantenimiento predictivo.

\section{EMISIONES ACÚSTICAS}

El método consiste en la medición de los niveles de intensidad del sonido, es decir, la detección de las ondas elásticas producidas por la aparición o crecimiento de un defecto en un material y conversión de estas ondas a señales eléctricas. Para ello se utilizan sensores basados en transductores piezoeléctricos, conectados directamente al transformador. La instrumentación de la Emisión
Acústica (EA) debe proporcionar alguna medida de la cantidad total de la emisión descubierta para realizar las correlaciones y determinar las fallas. Este método aplica para mediciones en fábrica (off-line) y en sitio donde se encuentra instalado el transformador (on-line). Generalmente las emisiones de ruido derivadas de los transformadores, provienen de tres clases de fuentes: del núcleo por efecto de la magnetostricción, de la corriente que circula por los devanados en presencia de un campo magnético y de equipos externos (ventiladores, bombas de recirculación e intercambiadores de calor) [119]. También pueden existir otras causas que originan un ruido anormal tales como descargas estáticas, resonancias, defectos estructurales o esfuerzos de cortocircuito durante fallas [23, 120]. Un ejemplo de la simulación resultante utilizando la técnica de emisiones acústicas aplicada a un transformador de potencia es mostrado en la Figura 5.

\section{Breve historia}

El origen de este método es atribuido a J. Kaiser en 1950, este hombre fue el primero en utilizar instrumentación electrónica para registrar los sonidos audibles producidos por metales durante 
Fuente: [120].

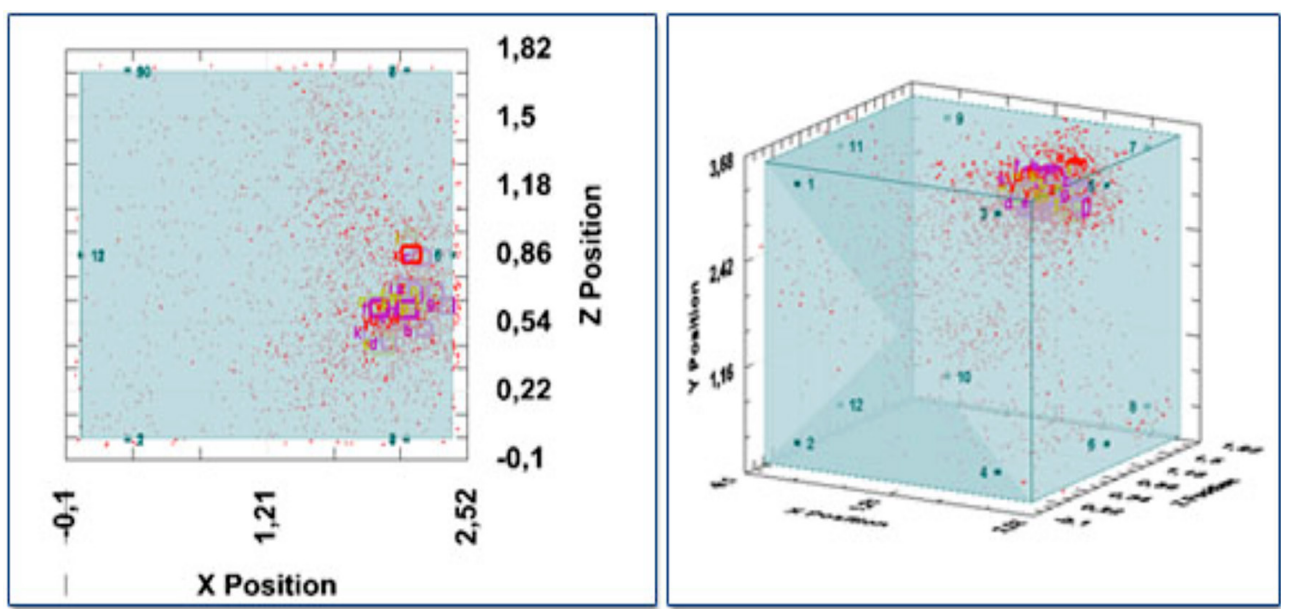

Figura 5. Simulación de resultados de una prueba de emisiones acústicas en un transformador.

su deformación, ocho años después se realizaron ciertas mejoras en la instrumentación por Schofield y Tatro. En 1967 se crea el grupo de trabajo AEWG (Acoustic Emission Working Group) a los que se les atribuye ser los precursores de aplicar el método de manera no invasiva.

En 1973 D. Allan y otros, publican artículo aplicando la prueba de EA a transformadores [121] y luego E. Howells, y E. Norton en 1978 publican un artículo relacionado con la detección de descargas parciales usando EA [122]. En los años posteriores, IEEE publica un estándar relacionado con la técnica de EA y la localización de fallas por descargas parciales [123]. En 2001 T. Boczar publica un estudio con cálculos para llevar los resultados de emisiones acústicas al dominio de la frecuencia y como resultado tener un espectro de frecuencia de las emisiones con el fin de hallar cierto tipo de descargas parciales [124]. Una década después, en el año 2014, se publica un estudio de A. Chicón y P. Berger [125] en donde se demuestra la potencialidad de utilizar EA para diagnosticar ciertos modos de falla del transformador en línea.

\section{ANÁLISIS COMPARATIVO DE LAS TÉCNICAS DE DIAGNÓSTICO}

Con el fin de analizar y clasificar las técnicas antes mencionadas, en esta sección se muestra la Tabla 3, basada en algunos criterios que a juicio de los autores son de especial relevancia y que comparten cada uno de los métodos de diagnóstico anteriormente descritos, permitiendo establecer un breve análisis cualitativo y cuantitativo según las principales características expuestas. Dichos criterios se describen a continuación:

- Aplicabilidad: se refiere únicamente a si la prueba es aplicada en la actualidad "en línea" o por "fuera de línea".

- Tipo de fallas detectables: hace mención a la naturaleza genérica de las fallas que pueden ser detectadas a través de la aplicación básica de la prueba.

- Máximo grado de confiabilidad: surge del grado de "certidumbre" ofrecido por los fabricantes en las fichas técnicas de los equipos que participan en las pruebas. Se ha tomado como referencia un promedio de dicho valor cuando se tienen varios equipos a nivel comercial ofrecidos para la ejecución de los ensayos.

- Normas técnicas y estándares internacionales: está basado en la normatividad más significativa alrededor del ensayo en mención, sin significar que sea de tipo exhaustivo, es decir, pueden existir otras normas alrededor del ensayo bajo estudio.

- Tipo de acceso a la muestra o medición: se basa en el hecho de la necesidad de apertura o desarme del transformador de potencia por parte del ensayo a realizar (Invasiva o No Invasiva) y si su aplicación es localizada directamente sobre el elemento bajo análisis o requiere llevar 
Tabla 3. Criterios de comparación cualitativa de las técnicas analizadas.

\begin{tabular}{|c|c|c|c|c|c|c|c|c|c|c|}
\hline$\stackrel{\Xi}{\Xi}$ & 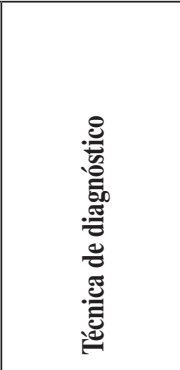 & 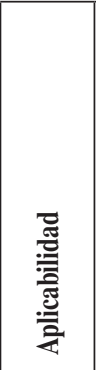 & 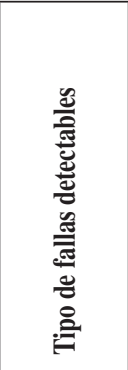 & 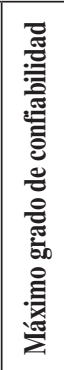 & 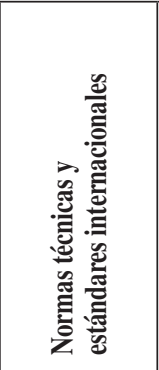 & 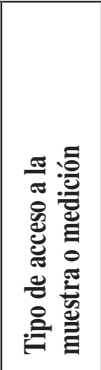 & 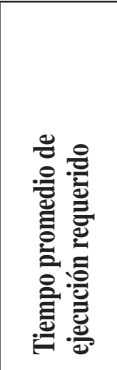 & 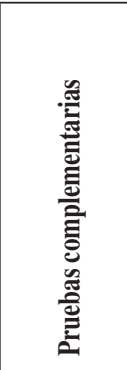 & 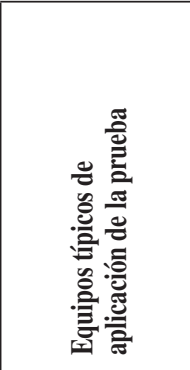 & 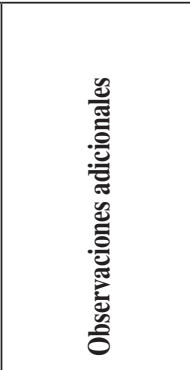 \\
\hline 1 & $\begin{array}{l}\text { Análisis de } \\
\text { Gases disueltos } \\
\text { (DGA) }\end{array}$ & On-line & $\begin{array}{l}\text { Eléctricas- } \\
\text { Térmicas }\end{array}$ & $85 \%$ & $\begin{array}{l}\text { No } \\
\text { estandarizado }\end{array}$ & \begin{tabular}{|l|} 
Directa- \\
No \\
invasiva \\
\end{tabular} & 5 horas & No & Transformadores. & $\begin{array}{l}\text { Puede conllevar } \\
\text { altos costos de } \\
\text { mantenimiento. }\end{array}$ \\
\hline 2 & $\begin{array}{l}\text { Análisis de } \\
\text { Respuesta en } \\
\text { Frecuencia } \\
\text { (FRA) }\end{array}$ & Off-line & $\begin{array}{l}\text { Mecánicas- } \\
\text { Eléctricas }\end{array}$ & $95 \%$ & $\begin{array}{l}\text { IEC 60076- } \\
\text { 18-IEEE } \\
\text { PC57.149 }\end{array}$ & $\begin{array}{l}\text { Directa- } \\
\text { No } \\
\text { invasiva }\end{array}$ & $20 \mathrm{~min}$. & \begin{tabular}{|l} 
Requiere \\
datos \\
históricos \\
de FRA
\end{tabular} & Transformadores. & \begin{tabular}{|l|} 
Generalmente, \\
requiere datos \\
de diseño y \\
construcción del \\
transformador. \\
\end{tabular} \\
\hline 3 & $\begin{array}{l}\text { Medición de } \\
\text { Corrientes de } \\
\text { Polarización y } \\
\text { Despolarización } \\
\text { (PDC) }\end{array}$ & Off-line & Eléctricas & $90 \%$ & \begin{tabular}{|l} 
IEC \\
C57.106- \\
IEC 61620
\end{tabular} & $\begin{array}{l}\text { Directa- } \\
\text { No } \\
\text { invasiva }\end{array}$ & 3 horas & FDS-RVM & $\begin{array}{l}\text { Transformadores, } \\
\text { Generadores, } \\
\text { Motores, } \\
\text { Capacitores. }\end{array}$ & $\begin{array}{l}\text { Presenta alta } \\
\text { susceptibilidad al } \\
\text { ruido eléctrico. }\end{array}$ \\
\hline 4 & $\begin{array}{l}\text { Espectroscopía } \\
\text { en el Dominio } \\
\text { de la frecuencia } \\
\text { (FDS) } \\
\end{array}$ & Off-line & Eléctricas & $90 \%$ & $\begin{array}{l}\text { IEEE } \\
\text { C57.106- } \\
\text { IEC 61620 }\end{array}$ & $\begin{array}{l}\text { Directa- } \\
\text { No } \\
\text { invasiva }\end{array}$ & 4 horas & PDC-RVM & $\begin{array}{l}\text { Transformadores, } \\
\text { Generadores, } \\
\text { Motores, } \\
\text { Capacitores. } \\
\end{array}$ & $\begin{array}{l}\text { Presenta alta } \\
\text { susceptibilidad a } \\
\text { la temperatura. }\end{array}$ \\
\hline 5 & $\begin{array}{l}\text { Medición de } \\
\text { Tensión de } \\
\text { Recuperación } \\
\text { (RVM) }\end{array}$ & Off-line & Eléctricas & $90 \%$ & $\begin{array}{l}\text { IEEE } \\
\text { C57.105- } \\
\text { IEC 61620 }\end{array}$ & $\begin{array}{l}\text { Directa- } \\
\text { No } \\
\text { invasiva }\end{array}$ & 2 horas & FDS-PDC & $\begin{array}{l}\text { Transformadores, } \\
\text { Capacitores. }\end{array}$ & $\begin{array}{l}\text { Presenta alta } \\
\text { susceptibilidad al } \\
\text { ruido eléctrico. }\end{array}$ \\
\hline 6 & $\begin{array}{l}\text { Análisis de } \\
\text { Furanos y } \\
\text { Grado de } \\
\text { Polimeización } \\
\text { (DP) }\end{array}$ & Off-line & $\begin{array}{l}\text { Químicas- } \\
\text { Térmicas }\end{array}$ & $93 \%$ & $\begin{array}{l}\text { ASTM } \\
\text { D5837 }\end{array}$ & $\begin{array}{l}\text { Directa- } \\
\text { No } \\
\text { invasiva }\end{array}$ & 2 horas & DP & Transformadores. & $\begin{array}{l}\text { Alta dependencia } \\
\text { del proceso de } \\
\text { muestreo. }\end{array}$ \\
\hline 7 & $\begin{array}{l}\text { Análisis } \\
\text { Termográfico } \\
\text { (IRT) }\end{array}$ & On-line & $\begin{array}{l}\text { Térmicas- } \\
\text { Mecánicas }\end{array}$ & $95 \%$ & \begin{tabular}{|l} 
IEEE \\
C57.119- \\
IEEE \\
C57.134
\end{tabular} & $\begin{array}{l}\text { Directa } \\
\text { - No } \\
\text { invasiva }\end{array}$ & Inmediato & $\begin{array}{l}\text { FDS-PDC- } \\
\text { RVM }\end{array}$ & $\begin{array}{l}\text { Transformadores, } \\
\text { Generadores, } \\
\text { Motores, } \\
\text { Capacitores y } \\
\text { Cables. }\end{array}$ & $\begin{array}{l}\text { Requiere conocer } \\
\text { previamente las } \\
\text { temperaturas } \\
\text { nominales de } \\
\text { operación de } \\
\text { los equipos para } \\
\text { diagnósticos. }\end{array}$ \\
\hline 8 & $\begin{array}{l}\text { Análisis de } \\
\text { Emisores } \\
\text { Acústicas (EA) }\end{array}$ & On-line & $\begin{array}{l}\text { Eléctricas- } \\
\text { Mecánicas }\end{array}$ & $95 \%$ & $\begin{array}{l}\text { IEC 60076- } \\
\text { 10-IEEE } \\
\text { C.57.12.90- } \\
\text { IEEE } \\
\text { C57.127 }\end{array}$ & $\begin{array}{l}\text { Directa } \\
\text { - No } \\
\text { invasiva }\end{array}$ & Inmediato & DGA-FRA & $\begin{array}{l}\text { Transformadores, } \\
\text { Generadores, } \\
\text { Motores, } \\
\text { Interruptores y } \\
\text { Bujes. }\end{array}$ & $\begin{array}{l}\text { Presenta } \\
\text { sustentabilidad a } \\
\text { ruidos externos. } \\
\text { Requiere el } \\
\text { historial de carga } \\
\text { del equipo a } \\
\text { diagnosticar. } \\
\end{array}$ \\
\hline
\end{tabular}

muestras para ser analizadas en laboratorio (por ejemplo los ensayos físico-químicos del aceite del transformador).

- Tiempo promedio de ejecución requerido: es solo un tiempo de referencia que surge de los ejemplos y casos mostrados en las referencias bibliográficas que describen cada una de las técnicas de diagnóstico a comparar.

- Pruebas complementarias: hace alusión a sí solo con la prueba en mención se tiene un resultado 
concluyente del elemento bajo análisis del transformador, o por el contrario, se recomienda complementar o contrastar la prueba con otras técnicas de diagnóstico.

- Equipos típicos de aplicación de las pruebas: destaca además de los transformadores de potencia, qué otros equipos se les puede aplicar típicamente la prueba en mención.

Como se puede observar en la Tabla 3, cada una de las técnicas estudiadas posee características específicas que la llevan a ser aplicada dependiendo del tipo de estudio que se requiera realizar al transformador. Sin embargo, si se pueden encontrar algunas características entre pruebas que pueden aportar un factor diferencial dentro de la escogencia de las mismas. Por ejemplo, únicamente tres de estas han podido ser aplicadas de manera "On-line" exitosamente (DGA, IRT y EA). Las otras técnicas, aún exigen la desenergización del transformador, convirtiéndose en un factor crítico y obligando a suspender los procesos de producción o transmisión de energía durante un tiempo considerable. Por último, se puede observar que muchas de las técnicas son aplicadas en conjunto o en forma complementaria, debido a que no existe una única técnica que permita detectar las fallas de todo tipo de naturaleza.

La gran mayoría presentan altos índices de confiabilidad (superiores al 90\%) dando una buena sensación de madurez y desarrollo de las técnicas. Ninguna es de tipo invasivo y tan solo una es realizada de manera indirecta (Análisis de Furanos y DP) por muestra de aceite. Otro aspecto interesante se refiere a los tiempos de ejecución, en donde hay una gran variabilidad dentro de un rango que va desde resultados casi inmediatos a resultados que pueden tomar hasta cinco horas de muestreo.

Además, los autores del presente estudio, también han querido presentar la Tabla 4, la cual es una comparación cuantitativa de las técnicas expuestas con aquellos factores que pueden ser de alto interés para los procesos de mantenimiento y gestión de activos. Dichos factores son la aplicabilidad, la confiabilidad y el tiempo de ejecución. En el caso de aplicabilidad se da un factor de "1" si es de tipo "On-line" o de "0" si ese de tipo "Off-line", en lo que respecta a la confiabilidad, sencillamente el factor de correspondencia es el porcentaje de la misma en términos decimales, y finalmente, el tiempo de ejecución viene dado por un factor mínimo de 0,25 para la técnica que requiere mayor tiempo, y un factor de 1,00 para la técnica de menor tiempo, las técnicas intermedias toman factores proporcionales a estos extremos.

La Tabla 4 deja ver de una forma numérica las posibles ventajas o desventajas de aplicación de las técnicas de diagnóstico. Es importante resaltar que dependiendo del tipo de falla que se desee encontrar, así mismo se tendrán unas técnicas de diagnóstico más idóneas que otras, tal es el caso de la humedad en el aislamiento celulósico del transformador, donde las pruebas por excelencia las cubren el FDS, PDC y RVM, sin embargo, ninguna de estas permite su aplicación en línea.

En muchos procesos productivos se decide recurrir a métodos indirectos tales como el análisis de Furanos o el DGA, que se basan en la condición físicoquímica del líquido dieléctrico (aceite mineral) para inferir el estado del aislamiento sólido al interior del

Tabla 4. Criterios de comparación cuantitativa de las técnicas analizadas.

\begin{tabular}{|c|l|c|c|c|}
\hline Ítem & \multicolumn{1}{|c|}{ Técnica de diagnóstico } & Aplicabilidad & Confiabilidad & Tiempo de ejecución \\
\hline 1 & Análisis de Gases Disueltos (DGA) & 1 & 0,85 & 0,25 \\
\hline 2 & Análisis de Respuesta en Frecuencia (FRA) & 0 & 0,95 & 0,95 \\
\hline 3 & $\begin{array}{l}\text { Medición de Corrientes de Polarización y } \\
\text { Despolarización (PDC) }\end{array}$ & 0 & 0,90 & 0,55 \\
\hline 4 & Espectroscopia en el Dominio de la Frecuencia (FDS) & 0 & 0,90 & 0,40 \\
\hline 5 & Medición de Tensión de Recuperación (RVM) & 0 & 0,90 & 0,70 \\
\hline 6 & Análisis de Furanos y Grado de Polimerización(DP) & 0 & 0,93 & 0,70 \\
\hline 7 & Análisis Termográfico (IRT) & 1 & 0,95 & 1,00 \\
\hline 8 & Análisis de Emisiones Acústicas (EA) & 1 & 0,95 & 1,00 \\
\hline
\end{tabular}


transformador, lo cual no necesariamente expresa la condición real del mismo; por ejemplo, la cantidad de agua porcentual contenida en este, la cual está altamente influenciada por dinámica de adsorción de humedad del aislamiento celulósico y su alta dependencia con la temperatura de operación del equipo.

La Figura 6 muestra un resumen con los datos de análisis cuantitativo de las técnicas según los criterios de comparación seleccionados.

\section{CONCLUSIONES}

En el presente trabajo, se llevó a cabo una revisión crítica con el fin de obtener información concreta sobre la evolución de ocho de las principales técnicas de diagnóstico y monitoreo de transformadores hasta el día de hoy, considerando las principales características que estas conllevan en su realización. En general, las técnicas estudiadas hacen uso de métodos no invasivos, evitando incluir costos operacionales por desacople de piezas o desarme total del transformador (vaciado de aceite o retiro de devanados o núcleo).

Algunos de estos métodos utilizan software y hardware especializados en el tratamiento de datos con el fin de obtener un análisis de mayor confiabilidad y de disminuir los tiempos de muestreo, tal es el caso de la Espectroscopia en el Dominio de la Frecuencia (FDS) o el Análisis de Gases Disueltos en línea (DGA on-line). Adicionalmente, para aquellas pruebas que son realizadas fuera de línea, se cuenta con la normatividad respectiva avalada por entidades internacionales como IEEE, IEC, ANSI, y CIGRÉ.

El análisis comparativo de los métodos de diagnóstico expuestos, permitió establecer que técnicas como la Termografía y Emisiones Acústicas tienen un alto potencial para ser integradas a sistemas de monitoreo en línea de transformadores de potencia. Adicionalmente, la técnica de DGA también presentó un buen comportamiento en este aspecto.

Finalmente, se espera que técnicas como FRA y FDS puedan realizarse en línea en los próximos años, reduciendo los costes de mantenimiento y aumentando la confiabilidad operativa de los transformadores de potencia.

\section{AGRADECIMIENTOS}

Los autores agradecen a la Universidad del Valle y al Departamento Administrativo de

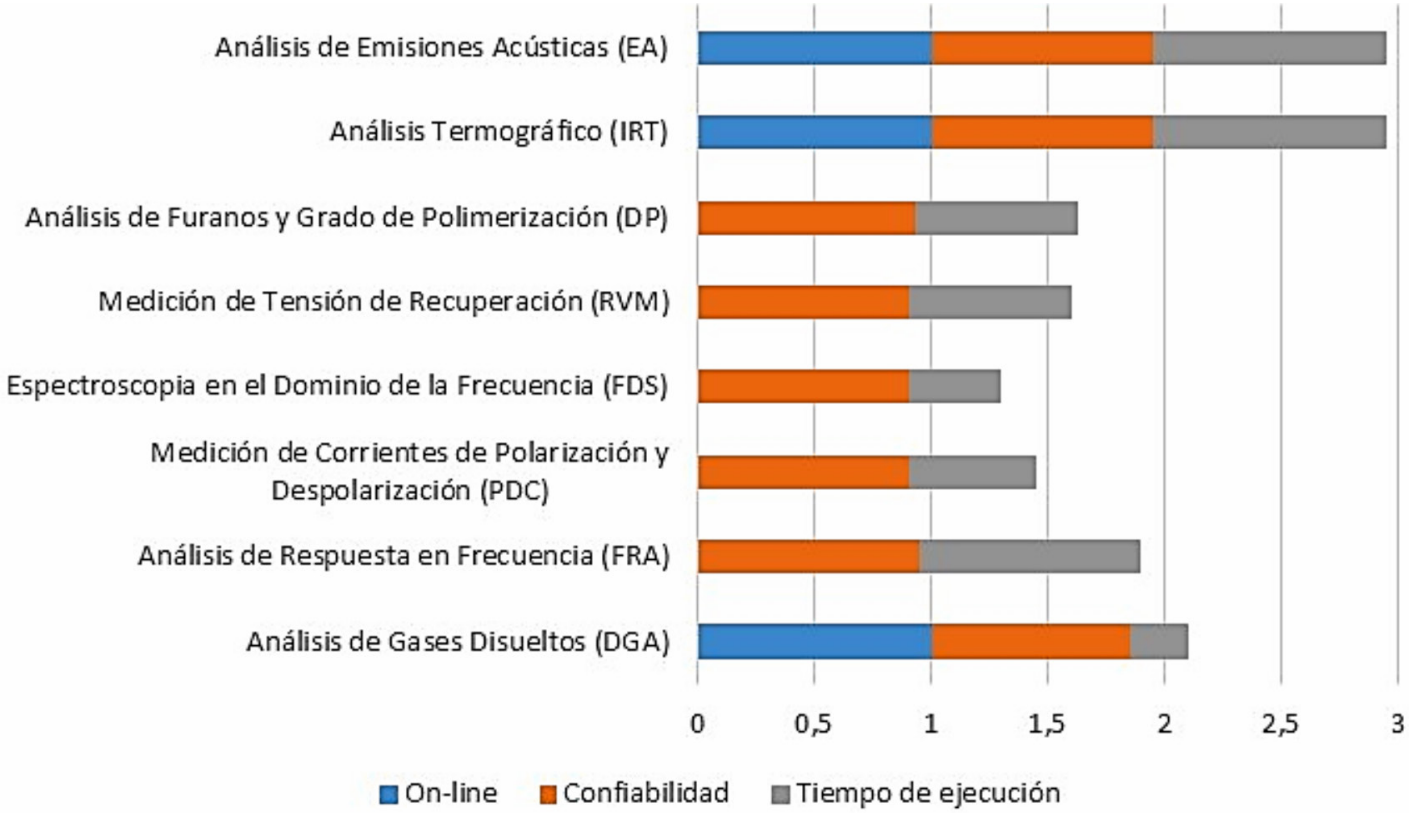

Figura 6. Resultados de la comparación cuantitativa de las técnicas. 
Ciencia, Tecnología e Innovación de Colombia, COLCIENCIAS, por apoyar este trabajo a través de los proyectos "Validación experimental de un modelo multifísico de difusión de humedadtemperatura para estimación de la dinámica de humedad en el aislamiento celulósico de los transformadores de potencia" código: 2857 y "Sistema de monitoreo en línea para la estimación de la vida útil de transformadores de potencia" código: 110671550932.

\section{REFERENCIAS}

[1] WEB. "Manual del usuario, operación y mantenimiento de transformadores de potencia”. Publicado por ABB Group. 2007. pp. 3-20. URL: https://library.e.abb.com/pu blic/9b7a293c90c90788852573fa007b78dc /1ZCL000002EG-ES_Manual\%20de1\%20 Usuario.pdf

[2] ASTMD3612 estándar. "Standard Test Method for Analysis of Gases Dissolved in Electrical Insulating Oil By Gas Chromatography". Vol. 4. ASTM Internacional. West Conshohocken, Estados Unidos. pp. 22. 2017. DOI: 10.1520/D3612-02R17.

[3] IEEE C57.146-2005 estándar. "IEEE Guide for the Interpretation of Gases Generated in Silicone-Immersed Transformers". Nueva York, Estados Unidos. 2006. DOI: 10.1109/ IEEESTD.2014.6966710.

[4] IEEEC57.104TM-2005 estándar. "IEEE Guide for the Interpretation of Gases Generated in Oil-Immersed Transformers". Segunda edición. Nueva York, Estados Unidos. 2008. DOI: 10.1109/IEEESTD.2006.99374.

[5] M. Horning, J. Kelly, S. Myers and R. Stebbins. "Transformer Maintenance Guide". S.D. Myers Inc. Tercera edición. Vol. 1, pp. 437. Ohio: Estados Unidos. ISBN-13: 978-0939320028. 2005.

[6] IEC 60599-2015 estándar. "Mineral oil-filled electrical equipment in service - Guidance on the interpretation of dissolved and free gases analysis". Primera edición. Vol. 1, pp. 78. Ginebra, Suiza. ISBN 978-2-83222899-9. 2015.

[7] M. Duval and A. DePablo. "Interpretation of gas-in-oil analysis using new IEC publication 60599 and IEC TC 10 databases". IEEE
Electrical Insulation Magazine. Vol. $17 \mathrm{~N}^{\circ} 2$, pp. 31-41. 2001. DOI: 10.1109/57.917529.

[8] M. Duval. "A review of faults detectable by gas-in-oil analysis in transformers". IEEE Electrical Insulation Magazine. Vol. 18, Issue 3, pp. 8-17. 2002. DOI: 10.1109/ MEI.2002.1014963.

[9] S.A. Ward. "Evaluating transformer condition using DGA oil analysis". 2003 Annual Report Conference on Electrical Insulation and Dielectric Phenomena, pp. 463-468. 2003. DOI: 10.1109/CEIDP.2003.1254893.

[10] M. Duval and J.J. Dukarm. "Improving the reliability of transformer gas-in-oil diagnosis". IEEE Electrical Insulation Magazine. Vol. 21, Issue 4, pp. 21-27. 2005. DOI: 10.1109/MEI.2005.1489986.

[11] Suwarno. "The Influence of arc on Dissolved Gases in Transformer Oils". 2006 IEEE 8th International Conference on Properties \& applications of Dielectric Materials. Bali. 2006, pp. 498-501. DOI: 10.1109/ ICPADM.2006.284224.

[12] M. Duval. "The Duval triangle for load tap changers, non-mineral oils and low temperature faults in transformers". IEEE Electrical Insulation Magazine. Vol. 24, Issue 6, pp. 22-29. 2008. DOI: 10.1109/ MEI.2008.4665347.

[13] ASTMD3612 estándar. "Standard Test Method for Analysis of Gases Dissolved in Electrical Insulating Oil by Gas Chromatography”. ASTM International. West Conshohocken, Estados Unidos, pp. 22. 2002. DOI: 10.1520/ D3612-02

[14] E.A. Mackenzie, J. Crossey, A. DePablo and W. Ferguson. "On-line monitoring and diagnostics for power transformers". 2010 IEEE International Symposium on Electrical Insulation. San Diego, Estados Unidos. 2010, pp. 1-5. DOI: 10.1109/ ELINSL.2010.5549734.

[15] M. Golkhah, S. Shamshirgar and M. Vahidi. "Artificial neural networks applied to DGA for fault diagnosis in oil-filled power transformers". Journal of Electrical and Electronics Engineering Research. Vol. 3, Issue 1, pp. 1-10. 2011. ISSN: 2141-2367.

[16] H.C. Sun, Y.C. Huang and C.M. Huang. "A Review of Dissolved Gas Analysis in Power Transformers". Energy Procedia. Vol. 14, 
Issue 1, pp. 1220-1225. 2012. ISSN: 18766102. DOI:10.1016/j.egypro.2011.12.1079.

[17] M. Duval and L. Lamarre. "The Duval Pentagon - A New Complementary Tool for the Interpretation of Dissolved Gas Analysis in Transformers". IEEE Electrical Insulation Magazine. Vol. 30, Issue 6, pp. 1-4. 2014. DOI: 10.1109/MEI.2014.6943428.

[18] J.T. Sarria, N.A. Guerrero y E. Rivas. "Estado del arte del análisis de gases disueltos en transformadores de potencia". Revista Facultad de Ingeniería. Universidad Distrital "Francisco José de Caldas". Vol. 23 No 36, pp. 105-122. 2014. eISSN 2357-5328.

[19] J.R. Secue, E.E. Mombello and C. V. Cardoso. "Review of Sweep Frequency Response Analysis SFRA for Assessment Winding Displacements and Deformation in Power Transformers". IEEE Latin America Transactions. Vol. 5, Issue 5, pp. 321-328. 2007. DOI: 10.1109/TLA.2007.4378524

[20] R. Breytenbach. "Winding Frequency Response Analysis Using the Impulse Frequency Response Analysis (IFRA) Method". IEEE FRA Specification. Starlogic IFRA Submission Version 1.0. Starlogic Instrument Development: South Africa, pp. 29. 2003.

[21] M. Gutten, M. Brandt, R. Polanský and P. Prosr. "SFRA Method - Frequency Analysis of Transformers". Measurement 2009. Proceedings of the 7th International Conference. Slovakia. pp. 369-372. 2009.

[22] A.L. Ortiz, M.A. Siqueira, J.C. Henning y N. Ramos. "Análisis de Fallas en Transformadores de Potencia tipos Secos y Propuesta de Ensayos Especiales para Evaluación de Condición". X seminario del sector eléctrico paraguayo - CIGRÉ, pp. 1-10. 2012.

[23] IEC 60076-10 estándar. "Power Transformers Part 10: determination of sound levels". Primera edición. Vol. 1, pp. 95. Ginebra, Suiza. ASIN: B000XYSDQG. 2005.

[24] International Chinese Standard DL 911/2004. "Frequency response analysis on winding deformation of power transformers". Segunda edición. República de China. 2016.

[25] J. Lapworth, T. Noonan, J. Christian and P. Picher. "Mechanical condition assessment of Transformer windings using Frequency
Response Analysis (FRA)”. Electra. № 228. 2006.

[26] Dick E.P. and Erven C.C. "Transformer Diagnostic Testing by Frequency Response Analysis". IEEE Transactions on Power Apparatus and Systems. Vol. PAS-97 N 6 , pp. 2144-2153. 1978.

[27] G. Kennedy, A. McGrail, and J. Lapworth. "Transformer sweep frequency response analysis (SFRA)". vEnergize. E-publishers. $\mathrm{N}^{\circ}$ October, pp. 1-12. 2007.

[28] IEEE C57.149-2012 estándar. "Guide for the Application and Interpretation of Frequency Response Analysis for Oil-Immersed Transformers". IEEE Std C57.149-2012.pp. 1-72.2013. DOI: 10.1109/ IEEESTD.2013.6475950.

[29] International Chinese Standard DL 911/2016. "Frequency Response Analysis on Winding deformation of power Transformers". Primera edición. República de China. 2005.

[30] IEC 60076-18:2012 estándar. "Power transformers - Part 18: Measurement of frequency response”. Vol. 1, pp. 90. Ginebra, Suiza. 2012.

[31] O. Aljohani, A. Abu-siada and S. Islam. "Impact of insulating oil degradation on the power transformer frequency response analysis". 2015 IEEE 11th International Conference on the Properties and Applications of Dielectric Materials (ICPADM). Sydney, NSW, pp. 396-399. 2015. DOI: 10.1109/ ICPADM.2015.7295292.

[32] O. Aljohani, A. Abu-Siada and Shengtao Li. "High frequency power transformer modelling for frequency response analysis studies". 2016 International Conference on Condition Monitoring and Diagnosis (CMD). Xi'an, pp. 291-294. 2016. DOI: 10.1109/ CMD.2016.7757818.

[33] Z. Zhao, S. Islam, N. Hashemnia, Di $\mathrm{Hu}$ and $\mathrm{C}$. Yao. "Understanding online frequency response signatures for transformer winding deformation: Axial displacement simulation”. 2016 International Conference on Condition Monitoring and Diagnosis (CMD). Xi' an, pp. 404-407. 2016. DOI: 10.1109/CMD.2016.7757845.

[34] N. Hashemnia, A. Abu-Siada and S. Islam. "Detection of Power Transformer Bushing Faults and Oil Degradation using Frequency 
Response Analysis". IEEE Transactions on Dielectrics and Electrical Insulation. Vol. 23, Issue 1, pp. 222-229. 2015. DOI: 10.1109/ TDEI.2015.005032.

[35] N.K. Wesley, S. Bhandari, A. Subramaniam, M. Bagheri and S.K. Panda. "Evaluation of statistical interpretation methods for frequency response analysis based winding fault detection of transformers". 2016 IEEE International Conference on Sustainable Energy Technologies (ICSET). Hanoi, pp. 36-41. 2016. DOI: 10.1109/ICSET.2016.7811753.

[36] M.H. Samimi and S. Tenbohlen. "FRA interpretation using numerical indices: State-of-the-art". International Journal of Electrical Power \& Energy Systems. Vol. 89, pp. 115-125. 2017. ISSN: 0142-0615.

[37] Y.Q. Sun et al. "Using impulse with appropriate repetition frequency in IFRA test to diagnosis winding deformation in transformer". 2016 IEEE International Conference on High Voltage Engineering and Application (ICHVE). Chengdu, pp. 1-4. 2016. DOI: 10.1109/ICHVE.2016.7800884.

[38] E. Gómez, G. Aponte, C. González y J. Pleite Guerra. "Current Status and Future Trends in Frequency-Response Analysis With a Transformer in Service". IEEE Transactions on Power Delivery. Vol. 28, Issue 2, pp. 1024-1031. 2013. DOI: 10.1109/ TPWRD.2012.2234141.

[39] E. Gómez, G. Aponte, J. Pleite, D. F. Silva y D. Hinestroza. "Application of Wavelet Transform to Obtain the Frequency Response of a Transformer From Transient Signals - Part 1: Theoretical Analysis". IEEE Transactions on Power Delivery. Vol. 28, Issue 3, pp. 1709-1714. 2013. DOI: 10.1109/ TPWRD.2013.2262058.

[40] E. Gómez, G. Aponte y J. Pleite. "Application of Wavelet Transform to Obtain the Frequency Response of a Transformer From Transient Signals - Part II: Practical Assessment and Validation". IEEE Transactions on Power Delivery. Vol. 29, Issue 5, pp. 2231-2238. 2014. DOI: 10.1109/TPWRD.2013.2295377.

[41] E. Gómez-Luna, G. Aponte y J. Pleite. "Sistema de obtención de la respuesta en frecuencia de máquinas eléctricas". Ingeniare. Revista chilena de ingeniería. Vol. 25, Issue 1, pp. 59-69. 2017.
[42] N. Muhamad, B.T. Phung y T.R. Blackburn. "Polarization and Depolarization Current (PDC) Tests on Biodegradable and Mineral Transformer Oils". Proceedings of the 16th International Symposium on High Voltage Engineering. pp. 1-6. 2009.

[43] T. Leibfried y A.J. Kachler. "Insulation diagnostics on power transformers using the polarisation and depolarisation current (PDC) analysis". Conference Record of the the 2002 IEEE International Symposium on Electrical Insulation. Boston. pp. 170-173. 2002. DOI: 10.1109/ELINSL.2002.995905.

[44] IEEE Recommended Practice for Testing Insulation Resistance of Electric Machinery. IEEE estándar 43-2013 (Revisión del estándar IEEE 43-2000). Vol. 1, pp. 37. 2014. DOI: 10.1109/IEEESTD.2014.6754111.

[45] IEEE Guide for Acceptance and Maintenance of Insulating Oil in Equipment. IEEE estándar C57.106-1991.pp. 1-25. 1992. DOI: 10.1109/ IEEESTD.1992.101092.

[46] IEEE Guide for Acceptance and Maintenance of Insulating Oil in Equipment. IEEE estándar C57.106-2002 (Revisión del estándar IEEE C57.106-1991).pp. 27. 2002. DOI: 10.1109/ IEEESTD.2002.94145.

[47] IEC 60422:2013 estándar. "Mineral insulating oils in electrical equipment - Supervision and maintenance guidance". Vol. 4, pp. 93. 2013.

[48] IEEE Guide for Diagnostic Field Testing of Electric Power Apparatus - Part 1: Oil Filled Power Transformers, Regulators, and Reactors. IEEE Std 62-1995. Vol. 1, pp. 68. 1995. DOI: 10.1109/IEEESTD.1995.122640.

[49] IEEE Guide for Diagnostic Field Testing of Fluid-Filled Power Transformers, Regulators, and Reactors. IEEE estándar C57.1522013. Vol. 2, pp. 121. 2013. DOI: 10.1109/ IEEESTD.2013.6544533.

[50] CIGRÉ. Working Group A2.30. Brochure 349. "Moisture equilibrium and moisture migration within Transformer insulation systems". 2008.

[51] V. der Houhanessian y W.S. Zaengl. "Onsite diagnosis of power transformers by means of relaxation current measurements". Conference Record of the 1998 IEEE International Symposium on Electrical Insulation. Arlington. Vol. 1, pp. 28-34. 1998. DOI: 10.1109/ELINSL.1998.704641. 
[52] J.J. Alff, V. Der Houhanessian, W.S. Zaengl y A.J. Kachler. "A novel, compact instrument for the measurement and evaluation of relaxation currents conceived for on-site diagnosis of electric power apparatus". Conference Record of the 2000 IEEE International Symposium on Electrical Insulation. Anaheim, Canadá. pp. 161-167. 2000. DOI: 10.1109/ELINSL.2000.845480.

[53] T. Kumar Saha. "Review of time-domain polarization measurements for assessing insulation condition in aged transformers". IEEE Transactions on Power Delivery. Vol. 18, Issue 4, pp. 1293-1301. 2003. DOI: 10.1109/TPWRD.2003.817741.

[54] T.K. Saha y P. Purkait. "Investigation of polarization and depolarization current measurements for the assessment of oilpaper insulation of aged transformers". IEEE Transactions on Dielectrics and Electrical Insulation. Vol. 11, Issue 1, pp. 144-154. 2004. DOI: 10.1109/TDEI.2004.1266329.

[55] H.A.P. Silva, W. Bassi y A.C.T. Diogo. "Noninvasive ageing assessment by means of polarization and depolarization currents analysis and its correlation with moisture content for power transformer life management". 2004 IEEE/PES Transmision and Distribution Conference and Exposition: Latin America. pp. 611-616. 2004. DOI: 10.1109/TDC.2004.1432449.

[56] T. Gradnik, M. Babuder y M. KoncanGradnik. "Estimation of water content in power transformers in service by polarization and depolarization current measurements". 2008 IEEE International Conference on Dielectric Liquids. FuturoscopeChasseneuil. pp. 1-4. 2008. DOI: 10.1109/ ICDL.2008.4622449.

[57] S.A. Bhumiwat. "Identification of overheating in transformer solid insulation by polarization depolarization current analysis". 2013 IEEE Electrical Insulation Conference (EIC). Ottawa. pp. 449-453. 2013. DOI: 10.1109/ EIC.2013.6554286.

[58] N.A. Muhamad, A.A. Suleiman, B.T. Phung y T.R. Blackburn. "Faults identification of biodegradable oil-filled transformers based on polarization and depolarization current measurement (PDC) method". IEEE Transactions on Dielectrics and Electrical
Insulation. Vol. 20, Issue 6, pp. 2299-2306. 2013. DOI: 10.1109/TDEI.2013.6678883.

[59] A.A. Suleiman, N. Bashir, N.A. Muhamad, N.A.M. Jamail y Q.E. Kamarudin. "Polarization \& depolarization current (PDC) measurement in cellulose insulating materials". 2013 Annual Report Conference on Electrical Insulation and Dielectric Phenomena. Shenzhen. pp. 1073-1076. 2013. DOI: 10.1109/CEIDP.2013.6748230.

[60] S.A. Bhumiwat. "Unusual conduction at oilpaper interface in transformers diagnosed by Polarization Depolarization Current (PDC) analysis". 2014 IEEE Electrical Insulation Conference (EIC). Philadelphia, Estados Unidos. pp. 185-188. 2014. DOI: 10.1109/ EIC.2014.6869372.

[61] M.A. Talib, N.A. Muhamad and Z.A. Malek. "Fault classification in power transformer using polarization depolarization current analysis". 2015 IEEE 11th International Conference on the Properties and Applications of Dielectric Materials (ICPADM). Sydney. pp. 983-986. 2015. doi: 10.1109/ ICPADM.2015.7295439.

[62] T.K. Saha, R. Middleton and A. Thomas. "Understanding frequency \& time domain polarisation methods for the insulation condition assessment of power transformers". 2009 IEEE Power \& Energy Society General Meeting. Calgary. pp. 1-8. 2009. DOI: 10.1109/PES.2009.5275851.

[63] N. Xavier, M. Izurieta, y A. Vásquez. "Pruebas de transformadores, espectroscopia en el dominio de la frecuencia y método de análisis de respuesta por barrido de frecuencia". Ingenius. $\mathrm{N}^{\circ}$ 5, pp. 55-62. 2011. ISSN-e 1390-860X.

[64] CIGRÉ. Brochure 414. "Dielectric Response Diagnoses For Transformer Windings". 2010.

[65] M. Ohlen. "Moisture in power transformers - How to estimate and what to do?". Water Energy Int. Vol. 70, Issue 12, pp. 13-17. 2013.

[66] IEC 61620 estándar. "Insulating liquids Determination of the dielectric dissipation factor by measurement of the conductance and capacitance - Test method". Vol. 1, pp. 43. Ginebra, Suiza. 1998.

[67] U. Gafvert, L. Adeen, M. Tapper, P. Ghasemi and B. Jonsson. "Dielectric 
spectroscopy in time and frequency domain applied to diagnostics of power transformers". Proceedings of the 6th International Conference on Properties and Applications of Dielectric Materials. Xi' an. Vol. 2, pp. 825-830. 2000. DOI: 10.1109/ ICPADM.2000.876357

[68] S.M. Gubanski et al. "Dielectric Response Methods for Diagnostics of Power Transformers". Paris, France: International Council on Large Electric Systems (CIGRE). 2002.

[69] W.S. Zaengl. "Applications of dielectric spectroscopy in time and frequency domain for HV power equipment". IEEE Electrical Insulation Magazine. Vol. 19, Issue 6, pp. 9-22. 2003. DOI: 10.1109/MEI.2003.1266348.

[70] W.S. Zaengl. "Dielectric spectroscopy in time and frequency domain for HV power equipment. I. Theoretical considerations". IEEE Electrical Insulation Magazine. Vol. 19, Issue 5, pp. 5-19. 2003. DOI: 10.1109/ MEI.2003.1238713.

[71] T.K. Saha and P. Purkait. "Investigations of Temperature Effects on the Dielectric Response Measurements of Transformer Oil-Paper Insulation System". IEEE Transactions on Power Delivery. Vol. 23, Issue 1, pp. 252-260. 2008. DOI: 10.1109/ TPWRD.2007.911123.

[72] G.K. Supramaniam, Z.F. Hussien and M. Aizam T. "Application of Frequency Domain Spectroscopy (FDS) in assessing dryness and ageing state of transformer insulation systems". 2008 IEEE 2nd International Power and Energy Conference. Johor Bahru, pp. 55-61. DOI: 10.1109/ PECON.2008.4762435.

[73] A. Bouaicha et al. "Dielectric spectroscopy techniques as quality control tool: a feasibility study”. IEEE Electrical Insulation Magazine. Vol. 25, Issue 1, pp. 6-14. 2009. DOI: 10.1109/ MEI.2009.4795464.

[74] I. Fofana et al. "On the frequency domain dielectric response of oil-paper insulation at low temperatures". IEEE Transactions on Dielectrics and Electrical Insulation. Vol. 17, Issue 3, pp. 799-807. 2010. DOI: 10.1109/ TDEI.2010.5492253.

[75] W. Wang, Y. Liu, J. Bi, Y. Gong and Y. Deng. "Effect Factors Analysis of Frequency
Domain Spectroscopy Test”. 2012 AsiaPacific Power and Energy Engineering Conference. Shanghai, pp. 1-5. 2012. DOI: 10.1109/APPEEC.2012.6307328.

[76] M.K. Pradhan and K.J.H. Yew. "Experimental investigation of insulation parameters affecting power transformer condition assessment using frequency domain spectroscopy". IEEE Transactions on Dielectrics and Electrical Insulation. Vol. 19, Issue 6, pp. 1851-1859. 2012. DOI: 10.1109/TDEI.2012.6396940.

[77] R. Liao, J. Hao, G. Chen and L. Yang. "Quantitative analysis of ageing condition of oil-paper insulation by frequency domain spectroscopy". IEEE Transactions on Dielectrics and Electrical Insulation. Vol. 19, Issue 3, pp. 821-830. 2012. DOI: 10.1109/ TDEI.2012.6215085.

[78] M. Jaya, Y. Rink y T. Leibfried. "Adapting frequency domain spectroscopy as an online monitoring tool for the insulation of power transformers". 2012 IEEE International Conference on Condition Monitoring and Diagnosis. Bali. pp. 501-504. 2012. doi: 10.1109/CMD.2012.6416189.

[79] Y. Liu, T. Zhang y X. Zhang. "A new method for assessment condition of oil-paper insulation based on the characteristics of frequency domain spectroscopy". 2016 IEEE International Conference on High Voltage Engineering and Application (ICHVE). Chengdu. pp. 1-4. 2016. DOI: 10.1109/ ICHVE.2016.7800752.

[80] Jianzhuo Dai, M. Dong, Yang Li, Jierui Zhou y Fuxin Wen. "Influence of nanoparticle concentration on the frequency domain spectroscopy properties of transformer oilbased nanofluids". 2016 IEEE Conference on Electrical Insulation and Dielectric Phenomena (CEIDP). Toronto. pp. 587-590. 2016. DOI: 10.1109/CEIDP.2016.7785543.

[81] M.A. Talib et al. "Diagnosis of transformer insulation condition using recovery voltage measurements". Proceedings. National Power Engineering Conference, 2003. PECon 2003. pp. 329-332. 2003. DOI: 10.1109/ PECON.2003.1437467.

[82] U. Gafvert y E. Ildstad. "Modelling return voltage measurements of multi-layer insulation systems". Proceedings of 1994 4th International Conference on Properties 
and Applications of Dielectric Materials (ICPADM). Brisbane. Vol. 1, pp. 123-126. 1994. DOI: 10.1109/ICPADM.1994.413926.

[83] E. Ildstad, U. Gafvert y P. Tharning. "Relation between return voltage and other methods for measurements of dielectric response". Proceedings of 1994 IEEE International Symposium on Electrical Insulation. Pittsburgh. pp. 25-28. 1994. DOI: 10.1109/ELINSL.1994.401476.

[84] G. Csepes, I. Hamos, R. Brooks y V. Karius. "Practical foundations of the RVM (recovery voltage method for oil/paper insulation diagnosis)". 1998 Annual Report Conference on Electrical Insulation and Dielectric Phenomena. Atlanta. Vol. 1, pp. 345-355. 1998. DOI: 10.1109/CEIDP.1998.734015.

[85] M. Chen, S.L. Hu y Z.H. Mo. "Fault diagnosis model of oil-immersed transformer based on Return Voltage Measurement". 2008 China International Conference on Electricity Distribution. Guangzhou, China. pp. 1-5. 2008. DOI: 10.1109/CICED.2008.5211653.

[86] R. Patsch y J. Menzel. "Ageing and degradation of power transformers - how to interpret Return Voltage Measurements". 2008 International Symposium on Electrical Insulating Materials (ISEIM 2008). pp. 179-182. 2008. DOI: 10.1109/ISEIM.2008.4664447.

[87] S. Sarkar, T. Sharma, A. Baral, B. Chatterjee, D. Dey y S. Chakravorti. "An expert system approach for transformer insulation diagnosis combining conventional diagnostic tests and PDC, RVM data”. IEEE Transactions on Dielectrics and Electrical Insulation. Vol. 21, Issue 2, pp. 882-891. 2014. DOI: 10.1109/ TDEI.2013.004052.

[88] C.K. Dwivedi y M.B. Daigavane. "Evaluation of Moisture Content in Paper-Oil of Aged Power Transformer Using RVM". 2009 Second International Conference on Emerging Trends in Engineering \& Technology. Nagpur. pp. 470-475. 2009. DOI: 10.1109/ ICETET.2009.92.

[89] S. Sarkar, T. Sharma, A. Baral, B. Chatterjee, D. Dey y S. Chakravorti. "A new approach for determination of moisture in paper insulation of in-situ power transformers by combining polarization-depolarization current and return voltage measurement results". IEEE Transactions on Dielectrics and Electrical
Insulation. Vol. 20, Issue 6, pp. 2325-2334. 2013. DOI: 10.1109/TDEI.2013.6678886.

[90] S. Sarkar et al. "A novel methodology for onsite validation of RV measurement data". 2012 1st International Conference on Power and Energy in NERIST (ICPEN). Nirjuli. pp. 1-5. 2012. DOI: 10.1109/ICPEN.2012.6492313.

[91] Á. Szirmai and Z.Á. Tamus. "Modelling of dielectric processes in oil-paper insulation for replacement of Return Voltage Measurement". 2016 Conference on Diagnostics in Electrical Engineering (Diagnostika). Pilsen. pp. 1-4. 2016. DOI: 10.1109/ DIAGNOSTIKA.2016.7736490.

[92] IEC 61198 estándar. "Mineral insulating oils - Methods for the determination of 2-furfural and related compounds". Vol. 1, pp. 28. 1993.

[93] ASTM D5837 estándar. "Standard Test Method for Furanic Compounds in Electrical Insulating Liquids by High- Performance Liquid Chromatography (HPLC)". pp. 1-9, 2013. ASTM International. West Conshohocken, Estados Unidos. pp. 22. 2002. DOI: 10.1520/D5837-15.

[94] R.J. Heywood, A.M. Emsley and M. Ali. "Degradation of cellulosic insulation in power transformers . Part 1: Factors affecting the measurement of the average viscometric degree of polymerisation of new and aged electrical papers". Vol. 147, Issue 2, pp. 86-90. 2000. DOI: 10.1049/ip-smt:20000076.

[95] A.M. Emsley, X. Xiao, R.J. Heywood y M. Ali. "Degradation of cellulosic insulation in power transformers. Part 2: formation of furan products in insulating oil". IEE Proceedings - Science, Measurement and Technology. Vol. 147, Issue 3, pp. 110-114. 2000. DOI: $10.1049 / \mathrm{ip}-\mathrm{smt}: 20000259$.

[96] A.M. Emsley, X. Xiao, R.J. Heywood and M. Ali. "Degradation of cellulosic insulation in power transformers. Part 3: effects of oxygen and water on ageing in oil". IEE Proceedings - Science, Measurement and Technology. Vol. 147, Issue 3, pp. 115-119. 2000. DOI: 10.1049/ip-smt:20000021.

[97] A.M. Emsley, R.J. Heywood, M. Ali and X. Xiao. "Degradation of cellulosic insulation in power transformers .4. Effects of ageing on the tensile strength of paper". IEE Proceedings - Science, Measurement and 
Technology, Vol. 147, $\mathrm{N}^{\circ}$ 6, pp. 285-290, Nov 2000. DOI: 10.1049/ip-smt:20000644.

[98] CIGRÉ. Working Group D1.01.10. Brochure 323. "Ageing of cellulose in mineral-oil insulated transformers". 2007.

[99] R.A. Ghunem, A.H. El-Hag y K. Assaleh. "Prediction of furan content in transformer oil using artificial neural networks (ANN)". 2010 IEEE International Symposium on Electrical Insulation. San Diego. pp. 1-4. 2010. DOI: 10.1109/ELINSL.2010.5549731.

[100] I. Hohlein-Atanasova and R. Frotscher. "Carbon oxides in the interpretation of dissolved gas analysis in transformers and tap changers". IEEE Electrical Insulation Magazine. Vol. 26, Issue 6, pp. 22-26. 2010. DOI: 10.1109/MEI.2010.5599976.

[101] A. Abu-Siada, S. P. Lai y S. M. Islam. "A Novel Fuzzy-Logic Approach for Furan Estimation in Transformer Oil". IEEE Transactions on Power Delivery. Vol. 27, Issue 2, pp. 469-474. 2012. DOI: 10.1109/ TPWRD.2012.2186986.

[102] L. Cheim, D. Platts, T. Prevost and S. $\mathrm{Xu}$. "Furan analysis for liquid power transformers". IEEE Electrical Insulation Magazine. Vol. 28, Issue 2, pp. 8-21. 2012. DOI: 10.1109/MEI.2012.6159177.

[103] N. Das, A. Abu-Siada y S. Islam. "New approach to estimate furan contents in transformer oil using spectroscopic analysis". 2012 22nd Australasian Universities Power Engineering Conference (AUPEC). Bali, pp. 1-4. 2012. E-ISBN: 978-979-18847-2-3.

[104] T. Leibfried, M. Jaya, N. Majer, M. Schafer, M. Stach y S. Voss. "Postmortem Investigation of Power Transformers - Profile of Degree of Polymerization and Correlation With Furan Concentration in the Oil". IEEE Transactions on Power Delivery. Vol. 28, Issue 2, pp. 886-893. 2013. DOI: 10.1109/ TPWRD.2013.2245152.

[105] N. Das, A. Abu-Siada and S. Islam. "Impact of conducting materials on furan-spectral correlation of transformer oil". 2013 Australasian Universities Power Engineering Conference (AUPEC). Hobart, pp. 1-4. 2013. DOI: 10.1109/AUPEC.2013.6725477.

[106] J. Jalbert, S. Duchesne, E. Rodriguez, P. Tétreault y P. Collin. "Robust and sensitive analysis of methanol and ethanol from cellulose degradation in mineral oils". Journal of Chromatography A. Volume 1256, pp. 240-245. 2012. ISSN 0021-9673.

[107] CIGRÉ. "Condition Assessment (is there enough experience to consider $\mathrm{MeOH}$ as a new marker for early stage paper degradation in transformers?)". $\mathrm{N}^{\circ}$ 1, pp. 2-3. 2009.

[108] N. A. Bakar y A. Abu-Siada. "High Voltage Power Transformer Paper Insulation Assessment Techniques". High Voltage Maintenance Forum. Perth: IDC Technologies, pp. 1-11. 2013. URL: http://hdl.handle. net/20.500.11937/32800.

[109] E.M. Rodriguez, S. Duchesne, J. Jalbert y M. Ryadi. "Understanding ethanol versus methanol formation from insulating paper in power transformers". Cellulose. Vol. 22, Issue 5, pp. 3225-3236. 2015. DOI: 10.1007/ s10570-015-0693.

[110] M. Concetta B, R. Maina, C. Sarzanini and V. Tumiatti. "GC Methods for Determination of Methanol and Ethanol in insulating mineral Oils as markers of cellulose degradation in power transformers". Chromatographia. No 77, pp. 1081-1089. 2014. DOI: 10.1007/ s10337-014-2650-8.

[111] M. Weiping, C. Fangxiao, S. Ying, X. Chungui y A. Ming. "Fault Diagnosis On Power Transformers Using Non-electric Method". 2006 International Conference on Power System Technology. Chongqing. pp. 1-5. 2006. DOI: 10.1109/ICPST.2006.321558.

[112] Manual. FLIR Systems AB. "Guía de termografía para mantenimiento predictivo", pp. 45, 2011. URL: www.flirmedia. com/MMC/THG/Brochures/T820264/ T820264_ES.pdf

[113] C. Meola. "Infrared Thermography Recent Advances and Future Trends". Primera edición. Napoli: Bentham Science, pp. 3-28. 2012. DOI: 10.2174/97816080514341120101.

[114] IEEE estándar. "Guide for Determination of Hottest-Spot Temperature in Dry-Type Transformers". IEEE estándar C57.134-2013 (Revisión del estándar IEEE C57.134-2000). Vol. 2, pp. 1-25. 2014. DOI: 10.1109/ IEEESTD.2014.6712026.

[115] IEEE estándar. "Recommended Practice for Performing Temperature Rise Tests on Oil-Immersed Power Transformers at Loads Beyond Nameplate Ratings". IEEE estándar 
C57.119-2001. Vol. 1, pp. 1-47. 2002. DOI: 10.1109/IEEESTD.2002.93571.

[116] N.Y. Utami, Y. Tamsir, A. Pharmatrisanti, H. Gumilang, B. Cahyono and R. Siregar. "Evaluation condition of transformer based on infrared thermography results". 2009 IEEE 9th International Conference on the Properties and Applications of Dielectric Materials. Harbin. pp. 1055-1058. 2009. DOI: 10.1109/ICPADM.2009.5252449.

[117] Q. Khan, A.A. Khan y F. Ahmad. "Condition monitoring tool for electrical equipment - Thermography”. 2016 International Conference on Electrical, Electronics, and Optimization Techniques (ICEEOT). Chennai. pp. 2802-2806. 2016. DOI: 10.1109/ ICEEOT.2016.7755208.

[118] FLIR Systems AB. "The Ultimate Infrared Handbook for R \& D Professionals". Segunda ed. 2012. URL: https://www.flirmedia. com/MMC/THG/Brochures/T559243/ T559243_EN.pdf

[119] IEEE estándar C57.12.90-2015. "IEEE Standard Test Code for Liquid-Immersed Distribution, Power, and Regulating Transformers". Vol. 1, pp. 1-13. 2015. DOI: 10.1109/IEEESTD.2016.7428800.

[120] Mistras south América. "Physical Acoustic South America". URL: http://www.pasa. com.br/espanhol/transformadores_trafo.asp.
[121] D.J. Allan, J.A.C. Forrest, E.L. Howitt and A.B. Petchell. "Electric and Acoustic Location of Discharges in Transformers". Conference on Diagnostic Testing of High Voltage Apparatus in Service. London, pp. 65-69. 1973.

[122] E. Howells and E.T. Norton. "Detection of Partial Discharges in Transformers Using Acoustic Emission Techniques". IEEE Transactions on Power Apparatus and Systems. Vol. PAS-97. № 5, pp. 1538-1549. 1978. DOI: 10.1109/TPAS.1978.354646.

[123] IEEE estándar. "Guide for the Detection and Location of Acoustic Emissions from Partial Discharges in Oil-Immersed Power Transformers and Reactors". IEEE estándar C57.127-2007 (Revisión del estándar IEEE C57.127-2000). Vol. 1, pp. 1-47. 2007.

[124] T. Boczar. "Identification of a specific type of PD from acoustic emission frequency spectra". IEEE Transactions on Dielectrics and Electrical Insulation. Vol. 8, Issue 4, pp. 598-606. 2001. DOI: 10.1109/94.946712.

[125] A. Cichoń and P. Berger. "Possibility of using acoustic emission method for testing load tap changers during normal operation of the transformer". 2014 ICHVE International Conference on High Voltage Engineering and Application. Poznan, pp. 1-4. 2014. DOI: 10.1109/ICHVE.2014.7035479. 